\title{
Evaluation of Insecticides against Pest Complex of Cashew
}

\author{
R. B. Patel, D. R. Patel, J. P. Makati and R. R. Patel
}

Dept. of Entomology, College of Agriculture, Navsari Agricultural University, Bharuch, Gujarat (392 012), India

\section{Corresponding Author}

R. B. Patel

e-mail: patel.devendra2829@yahoo.com
Article History

Article ID: IJEP227

Received in $27^{\text {th }}$ October, 2017

Received in revised form $8^{\text {th }}$ February, 2018

Accepted in final form $13^{\text {th }}$ February, 2018

\begin{abstract}
The investigation was carried out at the Agricultural experimental station, NAU, Paria (Gujarat, India) during 2009-10 to 2011-12. Total of eight treatments including control (unsprayed) were replicated thrice in randomized block design. Three sprays were carried out respectively at flushing, flowering and fruiting stages of cashew trees with the use of foot sprayer. Among all tested insecticides, lambda-cyhalothrin 5 EC @ 0.003\% recorded lowest incidence of cashew pest complex viz. tea mosquito bugs, Helopeltis antonii Sign., leaf minor, Acrocercops syngramma, inflorence webber, Lamida moncusalis and apple and nut borers Thylocoptila panrosema i.e. 0.82, 13.64, 12.77, 12.64 and $12.85 \%$, respectively and recorded the highest raw nut yield of $860 \mathrm{~kg} \mathrm{ha}^{-1}$ and it was at par with acetamiprid 20 SP @ $0.004 \%$.
\end{abstract}

Keywords: Borers, cashew, inflorescence webber, insecticide, leaf minor, bugs

\section{Introduction}

Cashew (Anacardium occidentale L.) is a very important foreign exchange earning crop of India. The estimated area under cashew in India is 8.55 lakh hectares and the production is around 5.73 lakh tons. The national average productivity is $815 \mathrm{~kg} \mathrm{ha}^{-1}$ (Maruthadurai et al., 2012). The pest infestation is a major constraint in cashew production. It is attacked by a number of insect pests during different stages of its growth and development. More than fifty species of insects are known to be infesting cashew in India in different degrees of intensity. However, when the extent of damage is taken into account only four are considered to be major pests. They are stem and root borer, tea mosquito bug, leaf miner, Apple and nut borer and responsible to cause crop loss. Tea mosquito attacks tender shoots and flower panicles and if the infestation is severe it causes yield reduction up to $30-40 \%$ (Devasahayam and Nair, 1986; Maruthadurai et al., 2012). Therefore, the experiment was conducted to test the efficacy of insecticides to control these pests under field condition.

\section{Materials and Methods}

The investigation was carried out at the Agricultural experimental station, NAU, Paria (Gujarat) during 200910 to 2011-12. Total of eight treatments including control (unsprayed) were replicated thrice in randomized block design. Three sprays were carried out respectively at flushing, flowering and fruiting stages of cashew trees with the use of foot sprayer. The time interval among sprays was of one month.

The damage grade caused by TMB was recorded before one day of each spray and again at 7 and 15 days after each spray. Damage scoring method for TMB in cashew score 0 to 4 is given viz., $0=$ no lesions or streak, $1=$ up to 3 necrotic lesions or streaks, 2=4-6 coalescing or non-coalescing lesions, 3 = above 6 coalescing or non-coalescing lesions, $4=$ lesions or streaks confluent complete drying of affected panicle or shoot. The damage leaf caused by leaf miner and inflorescence webber were counted separately from each lateral before one day of each spray and again at 15 days after each spray. Damage (\%) was work out. All the apples and nuts from the selected 52 leader shoots should be examined and damage (\%) caused by Apple and nut borers was worked out before one day of each spray and again at 15 days after each spray. The incremental cost benefit ratio (ICBR) was worked out on the basis of cost of various treatments including prevailing labour charges and market price of cashew fruits. Residual analysis of insecticides in cashew fruits was carried out at the Food Quality Testing Laboratory, Navsari Agricultural University, Navsari, Gujarat, India.

\section{Results and Discussion}

\subsection{Tea mosquito bugs, Helopeltis antonii}

The pooled data (Table 1 ) showed that seven day after spray the least infestation (0.96) were recorded in the treatment 


\begin{tabular}{|c|c|c|c|c|}
\hline \multirow{2}{*}{$\begin{array}{l}\text { Sr. } \\
\text { No. }\end{array}$} & \multirow[t]{2}{*}{ Treatments } & \multicolumn{3}{|c|}{ TMB damage rating (0-4) } \\
\hline & & Before Spray & After 7 days of Spray & After 15 days of Spray \\
\hline 1. & Acetamiprid 20 SP @ 0.004\% ; $0.2 \mathrm{~g} \mathrm{l}^{-1}$ & $1.20(0.96)$ & $0.96(0.45)$ & $0.82(0.17)$ \\
\hline 2. & Clothianiidin 50\% WDG @ 0.003\%; $0.6 \mathrm{ml} \mathrm{l}^{-1}$ & $1.20(0.98)$ & $0.99(0.50)$ & $0.86(0.26)$ \\
\hline 3. & Trizophos 40 EC @ 0.04\% ; $1 \mathrm{ml} \mathrm{l}^{-1}$ & $1.26(1.14)$ & $1.16(0.87)$ & $1.03(0.60)$ \\
\hline 4. & f-Cyhalothrin 5 EC @ 0.003\% ; $0.6 \mathrm{ml} \mathrm{l}^{-1}$ & $1.21(0.99)$ & $0.96(0.42)$ & $0.82(0.17)$ \\
\hline 5. & Profenophos 50EC @ 0.05\% ; 1 ml l-1 & $1.28(1.18)$ & $1.13(0.81)$ & $1.02(0.58)$ \\
\hline 6. & B-Cyfluthrin 200 SC @ 0.012\% ; $0.6 \mathrm{ml} \mathrm{l}^{-1}$ & $1.25(1.09)$ & $1.07(0.67)$ & $0.94(0.42)$ \\
\hline 7. & Endosulfan 35 EC @ 0.07\%; 2 ml l-1 & $1.26(1.12)$ & $1.13(0.81)$ & $0.99(0.52)$ \\
\hline \multirow[t]{6}{*}{8.} & Control & $1.42(1.56)$ & $1.51(1.83)$ & $1.46(1.90)$ \\
\hline & SEm \pm & 0.037 & 0.03 & 0.05 \\
\hline & $C D(p=0.05)$ & NS & 0.08 & 0.15 \\
\hline & CV\% & 5.94 & 7.62 & 6.88 \\
\hline & $\mathrm{SEm} \pm(T \times Y \times P)$ & 0.0432 & 0.03 & 0.05 \\
\hline & $\mathrm{CD}(p=0.05)$ & 0.121 & 0.08 & 0.15 \\
\hline
\end{tabular}

Figures in the parentheses are square root transformed values

of lambda-cyhalothrin 5 EC @ 0.003\% and acetamiprid 20 SP@ 0.004\%, however, they were statistically at par with clothianiidin 50 \% WDG @ 0.003\%. Similarly, at 15 days after sprays both the treatments viz. lambda-cyhalothrin 5 EC @ $0.003 \%$ and acetamiprid 20 SP @ 0.004\% recorded the lowest infestation (0.82), however, they were not significantly differ from Clothianiidin 50\% WDG and ß-Cyfluthrin 200 SC. The significant interaction effect $(T \times Y \times P)$ reveals that treatments behaved differently during different stages and years. These findings are in concurrence with the findings of Mahapatro (2008) and Bhat and Raviprasad (2007).

\subsection{Inflorence webber, Lamida moncusalis}

The lowest damage (12.77\%) was found in the treatment of lambda-cyhalothrin $5 \mathrm{EC}$ however, it was statistically at par with ß-cyfluthrin 200 SC (Table 2). The next best treatment was not significantly differing from the treatments of acetamiprid 20 SP, clothianiidin 50\% WDG and trizophos 40 EC. The non significant interaction effect $(T \times Y \times P)$ reveals that treatments behaved more or less similarly during different stages and years.

\subsection{Shoot tip caterpillar, Hypotima haligramma}

The significantly least damage (12.04\%) was recorded in the treatment of Lambda - Cyhalothrin 5 EC @ 0.003\%, however, it was followed by Acetamiprid 20 SP. The non significant interaction effect $(T \times Y \times P)$ reveals that treatments behaved more or less similarly during different stages and years (Table 2).

\subsection{Leaf minor, Acrocercops syngramma}

The treatment of lambda-cyhalothrin 5 EC @ 0.003\% had recorded the lowest damage (13.64\%). This treatment was statistically at par with ß-cyfluthrin 200 SC. The treatment of B-Cyfluthrin $200 \mathrm{SC}$ was not significantly differ from the treatments of acetamiprid 20 SP, clothianiidin 50\% WDG and profenophos 50EC. The non significant interaction effect $(T \times Y \times P)$ reveals that treatments behaved more or less similarly during different stages and years (Table 2).

\subsection{Apple and nut borers, Thylocoptila panrosema}

The least infestation (10.87) was found in the treatment of lambda-cyhalothrin $5 \mathrm{EC}$ and it was followed by B-Cyfluthrin 200 SC. The non significant interaction effect $(T \times Y \times P)$ reveals that treatments behaved more or less similarly during different stages and years (Table 2).

\subsection{Raw nut yield}

The treatment of lambda -cyhalothrin 5 EC ranked first by recording highest nut yield of $860 \mathrm{~kg} \mathrm{ha}^{-1}$ and it was statistically at par with Acetamiprid 20 SP and B-Cyfluthrin 200 SC. The treatment of Acetamiprid $20 \mathrm{SP}$ ranked second $\left(773 \mathrm{~kg} \mathrm{ha}^{-1}\right)$ in regard to gaining higher nut yield. The significant interaction effect $(T \times Y \times P)$ reveals that treatments behaved differently during different stages and years.

\subsection{Economics}

The data on economics of various treatments are presented in Table 4. The gross realization of INR $28380 \mathrm{ha}^{-1}$ was found in the treatment of Lambda-Cyhalothrin $5 \mathrm{EC}$ with BCR of 1:4.86. The treatment of Acetamiprid $20 \mathrm{SP}$ has recorded the gross realization of INR $23595 \mathrm{ha}^{-1}$ with BCR of 1:3.68. This finding is in confirmation with findings of Manjunaik and Chakravarthy (2013). 
Table 2: Field efficacy of insecticides against inflorescence webber, Lamida moncusalis Wlk., Shoot tip caterpillar, Hypotima haligramma $\mathrm{M}$, leaf minor Acrocercops syngramma and apple and nut borers Thylocoptila panrosema

\begin{tabular}{|c|c|c|c|c|c|c|c|c|c|c|}
\hline \multirow{3}{*}{$\begin{array}{l}\text { Sr. } \\
\text { No. }\end{array}$} & \multirow{3}{*}{ Treatments } & \multicolumn{9}{|c|}{$\%$ Incidence due to } \\
\hline & & \multicolumn{2}{|c|}{ Inflorence webber } & \multicolumn{2}{|c|}{ Shoot tip caterpillar } & \multicolumn{2}{|c|}{ Leaf minor } & \multicolumn{2}{|c|}{ Apple and nut borers } & \multirow{2}{*}{$\begin{array}{r}\text { Yield } \\
\left(\mathrm{kg} \mathrm{ha}^{-1}\right)\end{array}$} \\
\hline & & $\begin{array}{l}\text { Before } \\
\text { Spray }\end{array}$ & $\begin{array}{l}\text { After } \\
\text { spray }\end{array}$ & $\begin{array}{l}\text { Before } \\
\text { Spray }\end{array}$ & $\begin{array}{l}\text { After } \\
\text { spray }\end{array}$ & $\begin{array}{l}\text { Before } \\
\text { Spray }\end{array}$ & $\begin{array}{l}\text { After } \\
\text { spray }\end{array}$ & $\begin{array}{l}\text { Before } \\
\text { Spray }\end{array}$ & $\begin{array}{l}\text { After } \\
\text { spray }\end{array}$ & \\
\hline 1. & $\begin{array}{l}\text { Acetamiprid 20 SP @ } \\
0.004 \% ; 0.2 \mathrm{~g} \mathrm{l}^{-1}\end{array}$ & $\begin{array}{c}19.35 \\
(11.13)\end{array}$ & $\begin{array}{l}15.64 \\
(7.47)\end{array}$ & $\begin{array}{l}15.93 \\
(7.67)\end{array}$ & $\begin{array}{l}13.73 \\
(6.02)\end{array}$ & $\begin{array}{c}19.60 \\
(11.53)\end{array}$ & $\begin{array}{l}16.24 \\
(8.16)\end{array}$ & $\begin{array}{l}16.85 \\
(8.55)\end{array}$ & $\begin{array}{l}13.42 \\
(5.95)\end{array}$ & 773 \\
\hline 2. & $\begin{array}{l}\text { Clothianiidin } 50 \% \\
\text { WDG @ } 0.003 \% ; 0.6 \\
\mathrm{mll}^{-1}\end{array}$ & $\begin{array}{l}18.98 \\
(10.86)\end{array}$ & $\begin{array}{l}15.71 \\
(8.01)\end{array}$ & $\begin{array}{l}16.86 \\
(8.60)\end{array}$ & $\begin{array}{l}15.32 \\
(7.31)\end{array}$ & $\begin{array}{c}19.38 \\
(11.31)\end{array}$ & $\begin{array}{l}15.80 \\
(7.97)\end{array}$ & $\begin{array}{l}17.46 \\
(9.17)\end{array}$ & $\begin{array}{l}13.96 \\
(6.45)\end{array}$ & 725 \\
\hline 3. & $\begin{array}{l}\text { Trizophos } 40 \text { EC @ } \\
0.04 \% ; 1 \mathrm{ml}^{-1}\end{array}$ & $\begin{array}{c}19.71 \\
(11.63)\end{array}$ & $\begin{array}{l}15.92 \\
(7.97)\end{array}$ & $\begin{array}{l}17.35 \\
(8.83)\end{array}$ & $\begin{array}{l}15.25 \\
(7.45)\end{array}$ & $\begin{array}{c}20.11 \\
(12.03)\end{array}$ & $\begin{array}{l}16.68 \\
(8.57)\end{array}$ & $\begin{array}{l}17.10 \\
(8.84)\end{array}$ & $\begin{array}{l}15.13 \\
(7.17)\end{array}$ & 587 \\
\hline 4. & $\begin{array}{l}\text { f-Cyhalothrin } 5 \mathrm{EC} \\
@ 0.003 \% ; 0.6 \mathrm{ml} \mathrm{l}^{-1}\end{array}$ & $\begin{array}{c}18.37 \\
(10.09)\end{array}$ & $\begin{array}{l}12.77 \\
(5.38)\end{array}$ & $\begin{array}{l}15.00 \\
(6.89)\end{array}$ & $\begin{array}{l}12.04 \\
(4.75)\end{array}$ & $\begin{array}{c}19.56 \\
(11.39)\end{array}$ & $\begin{array}{l}13.64 \\
(6.03)\end{array}$ & $\begin{array}{l}16.48 \\
(8.24)\end{array}$ & $\begin{array}{l}10.87 \\
(4.43)\end{array}$ & 860 \\
\hline 5. & $\begin{array}{l}\text { Profenophos 50EC } \\
@ 0.05 \% ; 1 \mathrm{ml} \mathrm{l}^{-1}\end{array}$ & $\begin{array}{c}19.98 \\
(11.85)\end{array}$ & $\begin{array}{l}15.56 \\
(7.77)\end{array}$ & $\begin{array}{l}17.05 \\
(8.83)\end{array}$ & $\begin{array}{l}14.74 \\
(6.97)\end{array}$ & $\begin{array}{c}20.09 \\
(12.05)\end{array}$ & $\begin{array}{l}16.25 \\
(8.17)\end{array}$ & $\begin{array}{l}18.22 \\
(9.99)\end{array}$ & $\begin{array}{l}15.06 \\
(7.11)\end{array}$ & 554 \\
\hline 6. & $\begin{array}{l}\text { B-Cyfluthrin } 200 \mathrm{SC} \\
@ 0.012 \% ; 0.6 \mathrm{ml} \mathrm{l}^{-1}\end{array}$ & $\begin{array}{c}19.29 \\
(11.19)\end{array}$ & $\begin{array}{l}14.12 \\
(6.53)\end{array}$ & $\begin{array}{l}16.83 \\
(8.53)\end{array}$ & $\begin{array}{l}14.01 \\
(6.66)\end{array}$ & $\begin{array}{c}19.70 \\
(11.56)\end{array}$ & $\begin{array}{l}14.42 \\
(6.81)\end{array}$ & $\begin{array}{l}17.10 \\
(8.86)\end{array}$ & $\begin{array}{l}12.85 \\
(5.74)\end{array}$ & 755 \\
\hline 7. & $\begin{array}{l}\text { Endosulfan } 35 \text { EC @ } \\
0.07 \% ; 2 \mathrm{ml} \mathrm{l}^{-1}\end{array}$ & $\begin{array}{c}20.55 \\
(12.64)\end{array}$ & $\begin{array}{l}16.90 \\
(9.19)\end{array}$ & $\begin{array}{c}18.30 \\
(10.10)\end{array}$ & $\begin{array}{l}16.18 \\
(8.16)\end{array}$ & $\begin{array}{c}21.17 \\
(13.33)\end{array}$ & $\begin{array}{c}18.16 \\
(10.08)\end{array}$ & $\begin{array}{c}18.17 \\
(10.05)\end{array}$ & $\begin{array}{l}14.95 \\
(7.56)\end{array}$ & 524 \\
\hline 8. & Control & $\begin{array}{c}21.21 \\
(13.22)\end{array}$ & $\begin{array}{c}24.11 \\
(16.83)\end{array}$ & $\begin{array}{c}18.87 \\
(10.69)\end{array}$ & $\begin{array}{c}20.06 \\
(11.82)\end{array}$ & $\begin{array}{c}22.48 \\
(14.90)\end{array}$ & $\begin{array}{c}24.50 \\
(17.39)\end{array}$ & $\begin{array}{c}19.49 \\
(11.40)\end{array}$ & $\begin{array}{c}21.64 \\
(13.85)\end{array}$ & 344 \\
\hline & SEm \pm & 0.65 & 0.65 & 0.59 & 0.49 & 0.54 & 0.66 & 0.83 & 0.89 & 37.5 \\
\hline & $\mathrm{CD}(p=0.05)$ & NS & 1.82 & NS & 1.41 & NS & 1.84 & NS & 2.50 & 117 \\
\hline & $\mathrm{CV} \%$ & 0.65 & 1.09 & 0.98 & 0.94 & 0.79 & 1.12 & 0.79 & 1.21 & 26.6 \\
\hline & SEm $\pm(T \times Y \times P)$ & NS & NS & NS & NS & NS & NS & NS & NS & 77 \\
\hline & $C D(p=0.05)$ & 10.49 & 11.60 & 8.55 & 10.04 & 8.03 & 11.47 & 10.47 & 14.21 & 7.32 \\
\hline
\end{tabular}

Figures in the parentheses are square root transformed values

\subsection{Residual analysis of insecticides}

There were BMRL residue found in cashew fruit in best two insecticidal treatments i.e lambda-cyhalothrin 5 EC @ 0.003\% and acetamiprid 20 SP @ 0.004\% 15 days after insecticidal spraying as per the residual analysis of insecticides were carried out in the Food Quality Testing Laboratory, NAU, Navsari (Table 3 and 4).

\begin{tabular}{|c|c|c|c|}
\hline \multirow{2}{*}{$\begin{array}{l}\text { Sr. } \\
\text { No. }\end{array}$} & \multirow[t]{2}{*}{ Treatments } & \multicolumn{2}{|c|}{ No. of Beneficial } \\
\hline & & Before spray & After 7 days of spray \\
\hline 1. & Acetamiprid 20 SP @ 0.004\%; $0.2 \mathrm{~g} \mathrm{l}^{-1}$ & $1.97(3.67)$ & $1.72(2.67)$ \\
\hline 2. & Clothianiidin 50\% WDG @ 0.003\%; $0.6 \mathrm{ml} \mathrm{l}^{-1}$ & $1.62(2.00)$ & $1.03(0.67)$ \\
\hline 3. & Trizophos 40 EC @ 0.04\%; 1 ml l-1 & $1.76(2.88)$ & $1.03(0.67)$ \\
\hline 4. & f-Cyhalothrin 5 EC @ 0.003\%; $0.6 \mathrm{ml} \mathrm{l}^{-1}$ & $1.89(3.11)$ & $1.66(2.44)$ \\
\hline 5. & Profenophos 50EC @ 0.05\%; $1 \mathrm{ml} \mathrm{l}^{-1}$ & $1.88(3.11)$ & $1.06(0.78)$ \\
\hline 6. & B-Cyfluthrin 200 SC @ 0.012\%; 0.6 ml l-1 & $1.78(2.88)$ & $1.11(0.78)$ \\
\hline 7. & Endosulfan 35 EC @ 0.07\%; $2 \mathrm{ml} \mathrm{l}^{-1}$ & $2.02(3.33)$ & $1.38(1.44)$ \\
\hline
\end{tabular}




\begin{tabular}{llcc}
\hline Sr. & Treatments & \multicolumn{2}{c}{ No. of beneficial } \\
\cline { 3 - 4 } No. & & Before Spray & After 7 days of Spray \\
\hline 8. & Control & $1.78(2.88)$ & $2.02(4.00)$ \\
& SEm \pm & 0.21 & 0.10 \\
& CD $(p=0.05)$ & NS & 0.29 \\
& CV\% & 16.36 & 21.79 \\
\hline
\end{tabular}

Figures in the parentheses are square root transformed values

\begin{tabular}{|c|c|c|c|c|c|c|c|c|}
\hline $\begin{array}{l}\text { Sr. } \\
\text { No. }\end{array}$ & Treatments & PRNY & YIOC & QI & TC & $\mathrm{IIOC}$ & NP & $\mathrm{BCR}$ \\
\hline 1. & Acetamiprid 20 SP @ $0.004 \%$; $0.2 \mathrm{~g} \mathrm{l}^{-1}$ & 773 & 429 & 0.600 & 6405 & 23595 & 17190 & 1: 3.68 \\
\hline 2. & Clothianiidin 50\% WDG @0.003\%; $0.6 \mathrm{ml} \mathrm{l}^{-1}$ & 725 & 381 & 0.180 & 7446 & 20955 & 13509 & 1: 2.81 \\
\hline 3. & Trizophos 40 EC @ 0.04\% ; 1 ml l-1 & 587 & 243 & 3.000 & 6330 & 13365 & 7035 & 1: 2.17 \\
\hline 4. & f-Cyhalothrin 5 EC @ 0.003\% ; $0.6 \mathrm{ml} \mathrm{l}^{-1}$ & 860 & 516 & 1.800 & 5837 & 28380 & 22543 & 1: 4.86 \\
\hline 5. & Profenophos 50 EC @ 0.05\% ; $1 \mathrm{ml} \mathrm{l}^{-1}$ & 554 & 210 & 3.000 & 6576 & 11550 & 4974 & 1: 1.76 \\
\hline 6. & B-Cyfluthrin 200 SC @ 0.012\% ; $0.6 \mathrm{ml} \mathrm{l}^{-1}$ & 755 & 411 & 1.800 & 6330 & 22605 & 16275 & $1: 3.57$ \\
\hline 7. & Endosulfan 35 EC @ 0.07\%; 2 ml l-1 & 524 & 180 & 6.000 & 6552 & 9900 & 3348 & 1: 1.51 \\
\hline 8. & Control & 344 & -- & - & - & - & - & - \\
\hline
\end{tabular}

PRNY: Pooled raw nut yield $\left(\mathrm{kg} \mathrm{ha}^{-1}\right)$; YIOC: Yield increased over control ( $\left.\mathrm{kg} \mathrm{ha}^{-1}\right)$; QI: Quantity of insecticides $\left(\mathrm{kg} \mathrm{l}^{-1}\right.$ ha $\left.^{-1}\right) ; \mathrm{TC}^{-}$ Treatment cost (INR ha-1); IIOC: Income Increased over control (INR ha-1); NP: Net Profit (INR ha-1)

Prices of insecticides are as follow:

Acetamiprid 20 SP : INR 95 for 40 g pack; Profenophos 50EC: INR $532 \mathrm{I}^{-1}$; Clothianiidin 50\% WDG : INR 685 for $50 \mathrm{~g}$ pack; B-Cyfluthrin $200 \mathrm{SC}$ : INR $750 \mathrm{I}^{-1}$; Trizophos $40 \mathrm{EC}$ : INR $450 \mathrm{I}^{-1}$; Endosulfan $35 \mathrm{EC:}$ INR $262 \mathrm{I}^{-1}$; f-Cyhalothrin 5 EC: INR $476 \mathrm{I}^{-1}$; Price of Raw nut: INR $55 \mathrm{~kg}^{-1}$

\section{Conclusion}

From the three years pooled results it can be concluded that three sprays of Lambda-Cyhalothrin 5 EC @ 0.003\% (6 ml in 10 liter water) or Acetamiprid 20 SP @ 0.004\% (2 g in 10 I water) at flushing, flowering and fruiting were found most effective in recording low incidence of cashew pest complex as well as in gaining higher raw nut yield and cost-benefit ratio.

\section{References}

Bhat, P.S., Raviprasad, T.N., 2007. Management of tea mosquito bugs Helopeltis antonii Sign. With newer insecticides/products. National Seminar on Research Development and Marketting of Cashew, 20-21 $1^{\text {st }}$ Nov., 2007, 56.
Devasahayam, S., Nair, C.P.R., 1986 The tea mosquito bugs Helopeltis antonii Signorell on cashew in India. Journal of Plantation Crops 14(1), 1-10.

Mahapatro, G.K., 2008. Helopeltis management by chemicals in Cashew. Indian Journal of Entomology 70(4), 293-308.

Manjunaik, C., Chakravarthy, A.K., 2013. Sustainable management practices for tea mosquito bugs, Helopeltis antonii Signoret (Miriidae:Hemiptera) on Cashew. Karnataka Journal of Agricultural Science 26(1), 54-57.

Maruthadurai, R., Desai, A.R., Chidananda Prabhu, H.R., Singh, N.P., 2012. Insect-pests of Cashew and their management. Techanical Bulletin No. 28, ICAR Research Complex for Goa and Old-Goa, India, 1-16. 